International Journal of Modern Physics A

(C) World Scientific Publishing Company

\title{
The Minimal Type-II Seesaw Model and Flavor-dependent Leptogenesis
}

\author{
Shu Luo* and Zhi-zhong Xing \\ Institute of High Energy Physics, Chinese Academy of Sciences, Beijing 100049, China \\ *E-mail: luoshu@mail.ihep.ac.cn
}

Received October 25, 2018

\begin{abstract}
Current experimental data allow the zero value for one neutrino mass, either $m_{1}=0$ or $m_{3}=0$. This observation implies that a realistic neutrino mass texture can be established by starting from the limit (a) $m_{1}=m_{2}=0$ and $m_{3} \neq 0$ or (b) $m_{1}=m_{2} \neq 0$ and $m_{3}=0$. In both cases, we may introduce a particular perturbation which ensures the resultant neutrino mixing matrix to be the tri-bimaximal mixing pattern or its viable variations. We find that it is natural to incorporate this kind of neutrino mass matrix in the minimal Type-II seesaw model with only one heavy right-handed Majorana neutrino $N$. We show that it is possible to account for the cosmological baryon number asymmetry in the $m_{3}=0$ case via thermal leptogenesis, in which the CP-violating asymmetry of $N$ decays is attributed to the electron flavor.
\end{abstract}

Keywords: Minimal Seesaw; Leptogenesis; Flavor Effects.

PACS numbers: 11.30.Fs, 14.60.Pq, 14.60.St

\section{Introduction}

Recent neutrino oscillation experiments have provided us with convincing evidence that neutrinos are slightly massive and lepton flavors are not conserved. A global analysis of current neutrino oscillation data yields $\Delta m_{21}^{2}=(7.2 \cdots 8.9) \times 10^{-5} \mathrm{eV}^{2}$ and $\Delta m_{32}^{2}= \pm(2.1 \cdots 3.1) \times 10^{-3} \mathrm{eV}^{2}$ for three neutrino masses as well as $30^{\circ}<$ $\theta_{12}<38^{\circ}, 36^{\circ}<\theta_{23}<54^{\circ}$ and $\theta_{13}<10^{\circ}$ for three mixing angles at the $99 \%$ confidence level $!^{1}$ Three $\mathrm{CP}$-violating phases remain entirely unconstrained. Among many models or ansätze proposed to explain the smallness of neutrino masses, the seesaw mechanism ${ }^{2}$ seems to be most elegant and natural. Associated with the seesaw idea, the leptogenesis mechanism ${ }^{3}$ turns out to be an elegant and natural way to interpret the cosmological baryon number asymmetry which is characterized by $\eta_{B}=(6.1 \pm 0.2) \times 10^{-10} \underline{4}$

We find that at least two lessons can be learnt from current experimental data. First, the lightest neutrino is allowed to be massless; i.e., either $m_{1}=0$ (normal hierarchy) or $m_{3}=0$ (inverted hierarchy) has not been excluded. In both cases, the non-vanishing neutrino masses can be determined in terms of $\Delta m_{21}^{2}$ and $\left|\Delta m_{32}^{2}\right|$ : (a) if $m_{1}=0$, we have $m_{2}=\sqrt{\Delta m_{21}^{2}} \approx 8.94 \times 10^{-3} \mathrm{eV}, m_{3}=\sqrt{\left|\Delta m_{32}^{2}\right|+\Delta m_{21}^{2}} \approx$ $5.08 \times 10^{-2} \mathrm{eV}$; or (b) if $m_{3}=0$ we can obtain $m_{1}=\sqrt{\left|\Delta m_{32}^{2}\right|-\Delta m_{21}^{2}} \approx 4.92 \times$ 
$10^{-2} \mathrm{eV}$ and $m_{2}=\sqrt{\left|\Delta m_{32}^{2}\right|} \approx 5.00 \times 10^{-2} \mathrm{eV}$. Second, a special neutrino mixing pattern, the so-called tri-bimaximal mixing,, 5

$$
V=\left(\begin{array}{ccc}
2 / \sqrt{6} & 1 / \sqrt{3} & 0 \\
-1 / \sqrt{6} & 1 / \sqrt{3} & 1 / \sqrt{2} \\
1 / \sqrt{6} & -1 / \sqrt{3} & 1 / \sqrt{2}
\end{array}\right)
$$

is particularly favored. It yields $\theta_{12} \approx 35.3^{\circ}, \theta_{23}=45^{\circ}$ and $\theta_{13}=\rho=\sigma=0^{\circ}$. As a consequence of $\theta_{13}=0^{\circ}$, the Dirac phase $\delta$ is not well defined. In this talk, we shall first reconstruct the simplest neutrino mass texture for both mass hierarchies by combining the above two lessons and then discuss its seesaw realization and flavor-dependent leptogenesis.

\section{Deviations from Tri-bimaximal neutrino mixing}

Let us work in the basis where the charged-lepton mass matrix $M_{l}$ is diagonal. If $V$ is of the tri-bimaximal mixing pattern as given in Eq. (1), it can be decomposed into a product of two Euler rotation matrices: $V=O_{23} O_{12}$, where

$$
O_{12}=\left(\begin{array}{ccc}
\sqrt{2} / \sqrt{2+x^{2}} & x / \sqrt{2+x^{2}} & 0 \\
-x / \sqrt{2+x^{2}} & \sqrt{2} / \sqrt{2+x^{2}} & 0 \\
0 & 0 & 1
\end{array}\right), \quad O_{23}=\left(\begin{array}{ccc}
1 & 0 & 0 \\
0 & 1 / \sqrt{2} & 1 / \sqrt{2} \\
0 & -1 / \sqrt{2} & 1 / \sqrt{2}
\end{array}\right),
$$

with $x=1$. Allowing for small deviations of $x$ from 1 (or equivalently deviations of $\theta_{12}$ from $35.3^{\circ}$ ), we are then left with some variations of the tri-bimaximal neutrino mixing pattern which can fit current neutrino oscillation data if $0.82 \lesssim x \lesssim 1.10$.

Our strategy of reconstructing the neutrino mass matrix $M_{\nu}$ is three-fold: (1) we take a proper symmetry limit of $M_{\nu}$, denoted as $M_{\nu}^{(0)} \cdot M_{\nu}^{(0)}$ can be diagonalized by the transformation $O_{23}$ and the eigenvalues of $M_{\nu}^{(0)}$ satisfy (a) $m_{1}=m_{2}=0$ and $m_{3} \neq 0$ or (b) $m_{1}=m_{2} \neq 0$ and $m_{3}=0$; (2) we introduce a particular perturbation to $M_{\nu}^{(0)}$, denoted as $\Delta M_{\nu}$, which can be diagonalized by the transformation $\mathrm{O}_{23} \mathrm{O}_{12}$; (3) we require that $M_{\nu}=M_{\nu}^{(0)}+\Delta M_{\nu}$ should also be diagonalized by the transformation $O_{23} O_{12}$, and either $m_{1}=0$ or $m_{3}=0$ is guaranteed.

In the $m_{1}=0$ case, the neutrino mass matrix can be written as

$$
M_{\nu}=M_{\nu}^{(0)}+\Delta M_{\nu}=c\left[\left(\begin{array}{lll}
0 & 0 & 0 \\
0 & 1 & 1 \\
0 & 1 & 1
\end{array}\right)+\varepsilon\left(\begin{array}{ccc}
x^{2} & x & -x \\
x & 1 & -1 \\
-x & -1 & 1
\end{array}\right)\right] .
$$

Three neutrino masses turn out to be $m_{1}=0, m_{2}=\left(2+x^{2}\right) c \varepsilon$ and $m_{3}=2 c$.

In the $m_{3}=0$ case, we have

$$
M_{\nu}=M_{\nu}^{(0)}+\Delta M_{\nu}=c\left[\left(\begin{array}{ccc}
2 & 0 & 0 \\
0 & 1 & -1 \\
0 & -1 & 1
\end{array}\right)+\varepsilon\left(\begin{array}{ccc}
x^{2} & x & -x \\
x & 1 & -1 \\
-x & -1 & 1
\end{array}\right)\right],
$$

and three neutrino masses are $m_{1}=2 c, m_{2}=\left[2+\left(2+x^{2}\right) \varepsilon\right] c$ and $m_{3}=0$. 


\section{The minimal Type-II seesaw and $e$-leptogenesis}

Now let us consider how to derive the neutrino mass matrix $M_{\nu}$ in Eq. (3) or Eq. (4) from a specific seesaw model. Taking account of the fact that $M_{\nu}$ is composed of two mass matrices $M_{\nu}^{(0)}$ and $\Delta M_{\nu}$, we find that it is quite natural to incorporate $M_{\nu}$ in the minimal Type-II seesaw model with only one heavy right-handed Majorana neutrino $N \stackrel{6}{6}$ Comparing the well-known Type-II seesaw formula $M_{\nu} \simeq M_{L}-$ $M_{D} M_{R}^{-1} M_{D}^{T}$ with $M_{\nu}=M_{\nu}^{(0)}+\Delta M_{\nu}$, we arrive at

$$
M_{\nu}^{(0)}=M_{L}, \quad \Delta M_{\nu}=-M_{D} M_{R}^{-1} M_{D}^{T} .
$$

The texture of $\Delta M_{\nu}$ can be derived from Eq. (5) with a unique form of $M_{D}$, $M_{D}=i \sqrt{c \varepsilon M}(x, 1,-1)^{T}$, together with $M_{R}=M$ which is just the mass of the single right-handed Majorana neutrino. We remark that such a seesaw realization of the texture of $M_{\nu}$ does not involve any fine-tuning or cancellation.

We proceed to consider the leptogenesis in this minimal Type-II seesaw scenario. We allow $x$ to be complex in $M_{D}$ and its imaginary part is just responsible for CP violation in the model. This assumption surely modifies the pattern of the mixing matrix $V$ given in Section 2, but it maintains $\theta_{13}=0$ and $\theta_{23}=45^{\circ}$. 8 In the $m_{1}=0$ case, $M_{D}^{\dagger} M_{L} M_{D}=0$ holds, implying the absence of $\mathrm{CP}$ violation in the decays of $N$. Hence we shall focus our interest on the $m_{3}=0$ case in the following.

In the minimal Type-II seesaw model, the CP-violating asymmetry between $N \rightarrow l_{\alpha}+H^{\mathrm{c}}$ and its CP-conjugate process $N \rightarrow l_{\alpha}^{\mathrm{c}}+H$ arises from the interference between the tree-level amplitude and the $\Delta_{L}$-induced one-loop vertex correction, where $\Delta_{L}$ denotes the $S U(2)_{L}$ Higgs triplet. Since our numerical results indicate that the most favored range of $M$ is $10^{9} \mathrm{GeV} \sim 10^{11} \mathrm{GeV}$, we have to take account of the flavor effects on leptogenesis. ${ }^{9}$ For each lepton flavor $\alpha$, the corresponding $\mathrm{CP}$-violating asymmetry is approximately given by 10

$$
\epsilon_{\alpha} \simeq \frac{3 M}{16 \pi v^{2}} \cdot \frac{\sum_{\alpha, \beta} \operatorname{Im}\left[\left(M_{D}^{*}\right)_{\alpha 1}\left(M_{D}^{*}\right)_{\beta 1}\left(M_{L}\right)_{\alpha \beta}\right]}{\left(M_{D}^{\dagger} M_{D}\right)_{11}},
$$

where $v \equiv\langle H\rangle \simeq 174 \mathrm{GeV}$. In view of $M_{L}=M_{\nu}^{(0)}$ given in Eq. (4) together with $M_{D}=i \sqrt{c \varepsilon M}(x, 1,-1)^{T}$, we explicitly obtain

$$
\epsilon_{e}=-\frac{3 M c}{8 \pi v^{2}} \cdot \frac{\operatorname{Im}\left[\left(x^{*}\right)^{2}\right]}{2+|x|^{2}}, \quad \epsilon_{\mu}=\epsilon_{\tau}=0 .
$$

The overall CP-violating asymmetry turns out to be $\epsilon=\epsilon_{e}+\epsilon_{\mu}+\epsilon_{\tau}=\epsilon_{e}$. This result implies that only the $e$-flavor contributes to leptogenesis in our model.

The CP-violating asymmetry $\epsilon=\epsilon_{e}$ can partially be converted into a net baryon number asymmetry $\eta_{B} \simeq-0.96 \times 10^{-2} \sum_{\alpha} \epsilon_{\alpha} \kappa_{\alpha}=-0.96 \times 10^{-2} \epsilon_{e} \kappa_{e}$, where the efficiency factors $\kappa_{\alpha}$ measure the flavor-dependent washout effects 11 In the model under discussion, we have $\kappa_{e} \simeq(0.467 \cdots 0.64)$. The feasibility of producing $\eta_{B} \sim 6 \times 10^{-10}$ has been confirmed by our numerical analysis. 


\section{Summary}

In summary, we have proposed a new category of neutrino mass ansätze by starting from a combination of two phenomenological observations: (1) the lightest neutrino mass might be zero or vanishingly small, and (2) the neutrino mixing matrix might be the tri-bimaximal mixing pattern or a pattern close to it. We have incorporated the texture of $M_{\nu}$ in the minimal Type-II seesaw model and demonstrated that our model can simultaneously interpret current neutrino oscillation data and the cosmological baryon number asymmetry via thermal leptogenesis, in which only the electron flavor plays a role in the lepton-to-baryon conversion.

Finally let us remark that both the neutrino mass spectrum and the flavor mixing angles are well fixed and are stable against radiative corrections 12 in the proposed model. It is therefore easy to test them in the near future, when more accurate experimental data are available.

We would like to thank A. H. Chan and H. Fritzsch for collaboration 8 . This work was supported in part by the National Natural Science Foundation of China.

\section{References}

1. A. Strumia and F. Vissani, Nucl. Phys. B 726, 294 (2005); hep-ph/0606054.

2. P. Minkowski, Phys. Lett. B 67, 421 (1977); T. Yanagida, in Proceedings of the Workshop on Unified Theory and the Baryon Number of the Universe, edited by O. Sawada and A. Sugamoto (KEK, Tsukuba, 1979), p. 95; M. Gell-Mann, P. Ramond, and R. Slansky, in Supergravity, edited by P. van Nieuwenhuizen and D. Freedman (North Holland, Amsterdam, 1979), p. 315; S. L. Glashow, in Quarks and Leptons, edited by M. Lévy et al. (Plenum, New York, 1980), p. 707; R. N. Mohapatra and G. Senjanovic, Phys. Rev. Lett. 44, 912 (1980).

3. M. Fukugita and T. Yanagida, Phys. Lett. B 174, 45 (1986).

4. WMAP Collaboration, D. N. Spergel et al., arXiv:astro-ph/0603449

5. P. F. Harrison, D. H. Perkins and W. G. Scott, Phys. Lett. B 530, 167 (2002); Z. Z. Xing, Phys. Lett. B 533, 85 (2002); P. F. Harrison and W. G. Scott, Phys. Lett. B 535, 163 (2002); X. G. He and A. Zee, Phys. Lett. B 560, 87 (2003).

6. P. H. Gu, H. Zhang and S. Zhou, Phys. Rev. D 74, 076002 (2006).

7. J. Schechterm and J. W. F. Valle, Phys. Rev. D 22, 2227 (1980); M. Magg and C. Wetterich, Phys. Lett. B 94, 61 (1980); G. Lazarides, Q. Shafi and C. Wetterich, Nucl. Phys. B 181, 287 (1981)

8. A. H. Chan, H. Fritzsch, S. Luo and Z. Z. Xing, Phys. Rev. D 76, 073009 (2007).

9. See, e.g., R. Barbieri, P. Creminelli, A. Strumia and N. Tetradis, Nucl. Phys. B 575, 61 (2000); A. Abada, S. Davidson, F. X. Josse-Michaux, M. Losada and A. Riotto, JCAP 0604, 004 (2006); E. Nardi, Y. Nir, E. Roulet and J. Racker, JHEP 0601, 164 (2006); Z. Z. Xing and S. Zhou, Phys. Lett. B 653, 278 (2007).

10. S. Antusch, Phys. Rev. D 76, 023512 (2007).

11. W. Buchmüller, P. Di Bari and M. Plümacher, New J. Phys. 6, 105 (2004); G. F. Giudice, A. Notari, M. Raidal, A. Riotto and A. Strumia, Nucl. Phys. B 685, 89 (2004).

12. See, e.g., S. Antusch, J. Kersten, M. Lindner and M. Ratz, Nucl. Phys. B 674, 401 (2003); J. W. Mei and Z. Z. Xing, Phys. Rev. D 69, 073003 (2004); S. Luo and Z. Z. Xing, Phys. Lett. B 632, 341 (2006). 